\title{
I. Ueber Struvit von Homburg.
}

\author{
Von \\ Ernst Kalkowsky in Gotha.
}

(Mit Taf. I.)

In Homburg v. d. H. wurden im Hause des Herrn Reuter bei Gelegenheit eines Neubaues in der Dungergrube einige Krystalle von Struvit gefunden, von denen Herr Prof. Groth ungefähr ein Dutzend zugesendet erhielt; sie sind durchschnittlich $6-8 \mathrm{~mm}$ lang und $2-3 \mathrm{~mm}$ dick und unterliegen leicht der Verwitterung. Der Struvit zeigt eine merkwürdige Variabilităt des Habitus der Krystalle, und dieses Vorkommniss von Homburg weist wieder einen neuen Habitus auf, obwohl nur bereits bekannte Flächen erscheinen. Man kann die nach der Brachydiagonale gestreckten Krystalle ihrer Form nach mit der Spitze einer Stahlfeder vergleichen. Es erscheinen (Taf. I, Fig. 1) vorherrschend am oberen Ende das Doma $q=$ $\breve{P} \infty(011)$, am unteren Ende die Basis $c=0 P(00 \bar{T})$ und die Pyramide $t=$ $2 \breve{P} 2(12 T)$. Letztere Form tritt mit dem oberen Doma zu einer vierflächigen Spitze zusammen, meist aber nur an dem einen Ende der Brachydiagonale, während das andere Ende derselben mehr drusig ausgebildet ist; vielleicht waren die Krystalle damit aufgewachsen.

In der Zone des Brachydomas erscheint am unteren Ende der Krystalle gewöhnlich in schmalen Flächen das Doma $h=2 \breve{P} \infty(02 T)$; die Combinationskante zwiscben den beiden Brachydomen wird bisweilen durch das Brachypinakoid $b=\infty \breve{P} \infty(010)$ abgestumpft. In sehr kleinen oder schmalen Flächen erscheint mehrfach das Makrodoma $r=\bar{P} \infty(101)$ und nur an zwei Krystallen fand sich noch das Prisma $p=\infty \breve{P}_{2}(120)$.

Die Beschaffenheit der Krystallflächen gestattet nicht besonders gute Messungen, namentlich ist die Basis stets etwas gewölbt. Manche vergleichsweise untersuchten Hamburger Krystalle gaben weit schärfere Reflexe. An den Homburger Krystallen wurde das Axenverhältniss gefunden :

Groth, Zeitschrift f. Krystallogr. Xl.

$$
a: b: c=0,5685: 1: 0,9113,
$$


während Sa d eb e ck (Tschermak's min. Mitth. 1877, S. 173) an Hamburger Struviten fand:

$$
a: b: c=0,5667: 1: 0,9121 .
$$

An den Homburger Krystallen wurden die Winkel gefunden, wie folgt:

\begin{tabular}{|c|c|c|}
\hline$q: q=(011: 0 \overline{1} 1)$ & $\begin{array}{l}\text { Gemessen: } \\
={ }^{*} 84^{0} 41^{\prime}\end{array}$ & $\begin{array}{c}\text { Berechnet } \\
-\end{array}$ \\
\hline$q: b \rightleftharpoons(011: 010)$ & $47 \quad 6$ & $47039 \frac{1}{2}$ \\
\hline$q: h=(011: 02 \overline{1})$ & $76 \quad 12$ & 7625 \\
\hline$b: h=(010: 02 \pi)$ & 2838 & 2845 \\
\hline$t: t=(12 T: T 2 T)$ & $88 \quad 12$ & 8746 \\
\hline$t: q=(12 \overline{1}: 011)$ & 7932 & 7917 \\
\hline$r: r=(101: 10 \pi)$ & *63 55 & - \\
\hline$r: c=(10 \overline{1}: 00 \overline{1})$ & $57 \quad 47$ & $58 \quad 2 \frac{1}{2}$ \\
\hline
\end{tabular}

Auch in einigen anderen physikalischen Verhältnissen weicht der Homburger Struvit von den bisher bekannten Vorkommnissen etwas ab. Die Spaltbarkeit parallel der Basis und parallel dem Brachypinakoid tritt nur sehr unvollkommen hervor. Ebene der optischen Axen ist die Basis, erste Mittellinie ist die Makrodiagonale; die Doppelbrechung ist positiv; die Dispersion ist sehr kräftig, $v>\varrho$; der scheinbare Winkel der optischen Axen ist :

$$
\begin{aligned}
2 E & =59040^{\prime} \text { für die } L i \text {-Linie } \\
& =6056 \text { fur die } N a \text {-Linie. }
\end{aligned}
$$

Da es bereits bekannt ist, dass am Struvit die Basis bald am oberen, bald am unteren Ende der Krystalle auftritt, so die Frage $\mathrm{zu}$ entscheiden, ob die Homburger Krystalle wie in Fig. 1 oder umgekehrt zu stellen seien. Die Entscheidung konnte nur durch die thermoelektrischen Eigenschafter gegeben werden. Es wurden zunächst drei verschiedene Typen der Hamburger Struvite vermittelst der $\mathrm{K}$ un $\mathrm{dt}$ 'schen Bestäubungsmethode untersucht. Der Struvit hält eine Erwärmung auf $45^{\circ} \mathrm{C}$. recht wohl aus ohne jede Veränderung; die Versuche gelingen stets, wenn man die erwärmten Krystalle zunächst durch den Luftstrom aus dem langsam zusammengedrückten Bestäubungsapparat schnell abkühlt und dann sogleich möglichst schnell und stark mil dem Schwefel-Mennige-Gemisch uberstäubt. Die Versuche wurden bei verschiedenem Feuchtigkeitsgehalt der Luft mit demselben Erfolge wiederholt.

Bei der gewöhnlichsten Form der Hamburger Struvite (Fig. 2) zeigt sich stets der antiloge Pol an dem durch die Domen zugeschärften Ende der Krystalle; an den Kanten zeigt sich die stärkste elektrische Erregung. An dem vorherrschend durch die Basis begrenzten Ende liegt der analoge Pol, die negative Elektricität ist aber immer über einen grösseren Theil der Oberfläche vertheilt, als die positive. Eine stärkere Erregung an den Kan- 
ten zwischen Basis und Doma oder Pinakoid ist deutlich zu erkennen (Fig. 3). In der Mitte der Basis zeigte sich aber bei allen Versuchen ein kleines Feld mit positiver Elektricität, so dass es den Anschein hat, als wären die Krystalle in der Richtung der Verticalaxe nicht polar-thermoelektrisch. Wurde der obere Theil des Krystalles abgeschliffen, so dass nur eine von der naturlich unteren und einer kunstlichen oberen Basis begrenzte circa $1 \mathrm{~mm}$ dicke Tafel ubrig blieb, so zeigte die obere kunstliche Basis wieder ganz unzweifelhaft den antilogen Pol, während die naturliche Basis in unveränderter Weise an den Randkanten die stärkste Anbäufung negativer Elektricität und in der Mitte den Fleck mit positiver erkennen liess.

Es liegt hier wohl nur eine Influenzerscheinung vor, wie sie bei den Krystallen von dem Habitus wie Fig. 4 noch deutlicher hervortritt. Stellt man den antilogen Pol derselben wieder nach oben, so ist an diesen Krystallen die Basis am oberen Ende vorhanden, die Domen aber haben dieselben Indices wie in Fig. 2. Auch bei diesem Habitus dor Krystalle kommt die positive Erregung auf einem geringeren Theil der Oberfläche zum Vorschein, als die negative. Der analoge Pol tritt an der unteren scharfen Domenkante kräftig hervor, die Partien von negativer Elektricităt auf den Domenflächen 101 und $\bar{T} 01$ und auf den oberen Theilen der Brachypinakoide werden nach Form und Stärke nur durch influirende Kanten des antilogen Poles hervorgerufen.

Auf den Hamburger Krystallen, die nach dem Brachypinakoid flache, dreieckige Tafeln darstellen, Fig. 5 (Typus II, Subtypus, bei Sa d eb e ck), erscheint die positive Elektricität in grösserer Verbreitung an dem durch die schmale Basis und das Brachydoma begrenzten oberen Ende, der analoge Pol liegt auf dem unteren keilförmigen Ende, wo auf den Domenflächen die Elektricität stärker als auf dem Pinakoid auftritt.

Die Homburger Krystalle zeigten eine bedeutend geringere elektrische Erregbarkeit. Es ergab sich zunächst, dass auf den die Randkanten der Basis bildenden Flächen eine schmale Zone mit negativer Elektricität auftrat, wie Fig. 6 zeigt. Auf der Basis selbst, Fig. 7, ist diese Zone gegen das Centrum hin von ziemlich scharfen, den Randkanten parallelen Linien begrenzt, was augenscheinlich mit dem Auftreten eines schwach angedeuteten zonalen Aufbaues der Krystalle zusammenhängt. In der Mitte der Basis fand sich wieder ein kleines Feld mit positiver Elektricität, wenn dasselbe auch nur durch ein oder zwei Körnchen Schwefel sichtbar wurde.

Die Pyramidenflächen zeigten auf ihren oberen Theilen kaum Spuren einer elektrischen Erregung (vergl. Fig. 6, welche zugleich die gewöhnliche, flächenärmere Form der Krystalle veranschaulicht); auch auf den Flächen des Domas $q$ haftete nur wenig Staub: schräg gegen das Licht gehalten zeigten sie einen rothen Schein von Mennige. Da die Kante dieses Domas aber gerade die längste an den Krystallen ist, so konnte an ihr viel- 
leicht die elektrische Spannung nicht einen genügend hohen Grad erreichen, um nicht durch Influenzerscheinungen verdeckt zu werden. Es wurde deshalb durch Anschleifen von Flächen ungefähr von der Lage des oberen Makrodomas ein körperlich enger begrenzter Pol geschaffen, Fig. 8, und dieser erwies sich dann auch deutlich als antiloger Pol, namentlich wenn der Krystall mit einer Brachydomenfläche auf den als Träger dienenden Kork aufgelegt wurde; die nicht leitende Korksubstanz bält dann gleichsam die Elektricität fest, so dass die Reaction stärker hervortritt.

Stellt man die verschiedenen Typen der Struvite stets so auf, dass der antiloge Pol nach oben gerichtet ist, dann haben die Homburger Krystalle in Fig. 1 die richtige Stellung erhalten.

Die vorliegende sowie einige andere Untersuchungen gestattete mir Herr Professor Groth gutigst im mineralogischen Institut der Universität München auszuführen; hierfurr, wie für alle wäbrend meines Aufenthaltes in Munchen mir erwiesene Freundlichkeit spreche ich auch an dieser Stelle meinen ergebensten Dank aus.

\section{Erklärung der Tafel I.}

Fig. 1. Struvit von Homburg, flächenreichster Krystall.

Fig. 2. Struvit von Hamburg, gewöhnlichste Form mit $\bar{P} \infty(101), \breve{P} \infty(011)$ und $\infty \breve{P} \infty(01 \theta)$ oben und $\frac{1}{3} \bar{P} \infty(10 \overline{3})$ und $0 P(00 \bar{T})$ unten; gelb Verbreitung der positiven, roth der negativen Elektricität beim Abkühlen der Krystalle.

Fig. 3. Basis des Krystalls Fig. 2 mit centralem Feld von positiver Elektricität.

Fig. 4. Struvit von Hamburg mit den Flächen $0 P(001), \bar{P} \infty(101), \breve{p}_{\infty}(011)$ und $\infty \breve{P} \infty(010)$ oben und $\bar{P} \infty(10 \bar{T})$ und $\infty \breve{P} \infty(010)$ unten. Verbreitung der elektrischen Erregung.

Fig. 5. Struvit von Hamburg von dreieckig tafelförmigem Habilus, mit $\breve{P} \infty(011)$ und $0 P(001)$ oben und $\infty \breve{P} \infty(010)$ und $\vec{P} \infty(10 \bar{T})$ unten. Verbreitung der elektrischen Erregung.

Fig. 6. Einfachere Form des Struvits von Homburg, mit schwacher elektrischer Erregbarkeit.

Fig. 7. Verbreitung der Elektricität auf der Basis von Homburger Struviten.

Fig. 8. Dasselbe Individuum wie Fig. 6, mil angeschliffenen Polfächen und in Folge dessen deutlich hervortretendem antilogen Pol. 